MATCH4 molecule was constructed containing bivalent lowaffinity MSLN binding domains, a CD3 binding domain, and a serum albumin-binding domain for half-life extension. This molecule was tested in a cytotoxicity assay using human PBMCs co-cultured with H226 or MeT-5A cells, which express high or low levels of MSLN, respectively. The MeT5A line, derived from mesothelial cells in the pleural fluid of non-cancerous individuals, represents normal MSLN-expressing cells. Soluble MSLN was added to determine effects on cytotoxicity. In vivo xenograft mouse studies were conducted using a tumor cell/PBMC co-implantation model, followed by regular dosing with molecules of interest.

Results Here we report the design and the promising preclinical activity of the MATCH4 molecule in vitro and in vivo. We demonstrate that the low-affinity bivalent MSLN T cell engager has increased in vitro potency in $\mathrm{T}$ cell activation and tumor cell killing, as compared to a high-affinity monovalent counterpart on high MSLN expressing cells. We also demonstrate that the activity on low MSLN expressing cells is reduced for the low-affinity bivalent compared to the high affinity monovalent molecule. Because soluble MSLN is shed from cancer cells into cancer patient serum, we also demonstrate that up to $500 \mathrm{ng} / \mathrm{mL}$ of soluble MSLN does not interfere with the cytotoxic activity of the low affinity bivalent $\mathrm{T}$ cell engager, compared to the effects of soluble MSLN on a high affinity monovalent $\mathrm{T}$ cell engager. Importantly, we demonstrate in vivo that the low-affinity bivalent molecule significantly inhibits tumor growth in a dose-dependent manner.

Conclusions Collectively, these data demonstrate anti-tumor efficacy by this novel multispecific low affinity bivalent $\mathrm{T}$ cell engager. These data indicate the potential of this molecule to increase the therapeutic window by reducing safety concerns on normal tissue where MSLN expression is low, and yet increase cytotoxicity to MSLN-expressing cancer cells.

http://dx.doi.org/10.1136/jitc-2020-SITC2020.0684

\section{A HIGHLY SELECTIVE AND POTENT HPK1 INHIBITOR ENHANCES IMMUNE CELL ACTIVATION AND INDUCES ROBUST TUMOR GROWTH INHIBITION IN A MURINE SYNGENEIC TUMOR MODEL}

${ }^{1}$ David Ciccone*, ${ }^{2}$ Vad Lazari, ${ }^{2}$ lan Linney, ${ }^{2}$ Michael Briggs, ${ }^{1}$ Samantha Carreiro, ${ }^{1}$ Joshua McElwee, ${ }^{2}$ lan Waddell, ${ }^{2}$ Chris Hill, ${ }^{1}$ Christine Loh, ${ }^{1}$ Peter Tummino, ${ }^{1}$ Alan Collis, ${ }^{1}$ Neelu Kaila. 'Nimbus Therapeutics, Cambridge, MA, USA; ${ }^{2}$ Charles River Laboratories, Chesterford Research Park, UK

Background HPK1, a member of the MAP4K family of protein serine/threonine kinases, is involved in regulating signal transduction cascades in cells of hematopoietic origin. Recent data from HPK1 knockout animals and kinase-inactive knockin animals underscores the role of HPK1 in negatively regulating immune cell activation. This negative-feedback role of HPK1 combined with its restricted expression in cells of hematopoietic origin, make it a compelling drug target for enhancing anti-tumor immunity.

Methods A structure-based drug design approach was used to identify potent and selective inhibitors of HPK1. Biochemical assays, as well as primary human and mouse immune cellbased activation assays, were utilized for multiple iterations of structure-activity relationship (SAR) studies. In vivo efficacy, target engagement and pharmacodynamic data were generated using murine syngeneic tumor models.
Results A highly potent, HPK1 inhibitor was identified, that showed high selectivity against $\mathrm{T}$ cell-specific kinases and kinases in the MAP4K family. In vitro, HPK1 small molecule inhibition resulted in enhanced IL-2 production in primary mouse and human T cells, enhanced IL- 6 and IgG production in primary human B cells, and enhanced mouse dendritic cell activation and antigen presentation capacity. Furthermore, HPK1 inhibition alleviated the immuno-suppressive effects of PGE2 on naïve human $\mathrm{T}$ cells and restored the proliferative capacity of exhausted human $\mathrm{T}$ cells. In vivo, HPK1 inhibitionHPK1 inhibition abrogated $\mathrm{T}$ cell receptor-stimulated phospho-SLP-76, enhanced cytokine production, and mediated robust tumor growth inhibition in a murine syngeneic tumor model.

Conclusions Pharmacological blockade of HPK1 kinase activity represents a novel and potentially valuable immunomodulatory approach for anti-tumor immunity.

http://dx.doi.org/10.1136/jitc-2020-SITC2020.0685

\section{PRECLINICAL CHARACTERIZATION OF A NOVEL THERAPEUTIC ANTIBODY TARGETING LILRB2}

${ }^{1}$ Maria Jose Costa*, ${ }^{1}$ Ryan Stafford, ${ }^{2}$ Zhiqiang Ku, ${ }^{1}$ Jing-Tyan Ma, ${ }^{1}$ Krista McCutcheon, ${ }^{3}$ Xiaoye Liu, ${ }^{3}$ Heyu Chen, ${ }^{1}$ Kyu Hong, ${ }^{1}$ Tao Huang, ${ }^{2}$ Ningyan Zhang, ${ }^{2}$ Zhiqiang An, ${ }^{3}$ Cheng Cheng Zhang, ${ }^{1} X$ Charlene Liao, ${ }^{1}$ An Song. ${ }^{1}$ Immune-Onc Therapeutics, Palo Alto, CA, USA; ${ }^{2}$ University of Texas Health Science Center, Houston, TX, USA; ${ }^{3}$ University of Texas Southwestern, Dallas, TX, USA

Background Myeloid-derived suppressor cells and tumor-associated macrophages inhibit anti-cancer immune responses systemically and in the tumor microenvironment, respectively, thereby limiting the efficacy of immune checkpoint blockers. ${ }^{1-}$ 5 However, the plasticity of myeloid cells may enable therapeutic intervention. The inhibitory receptor LILRB2/ILT4, which is expressed primarily in myeloid cells (monocytes, macrophages, dendritic cells and neutrophils), has emerged as a key immune checkpoint mediating the tolerogenic activity of myeloid cells associated with cancer. ${ }^{6-8}$ LILRB2/ILT4 has several ligands (classical and non-classical MHC-I, ANGPTL2/5, SEMA4A and CD1) and most of these are known to contribute to immune suppression in the tumor microenvironment. ${ }^{9-}$ 14 Thus, LILRB2/ILT4 is a promising target to overcome protumor myeloid cell activity. IO-108 is a fully human IgG4 therapeutic candidate that binds LILRB2/ILT4 with high affinity and specificity, thereby blocking its ligand interactions.

Methods We used computational approaches to evaluate LILRB2/ILT4 expression in solid tumors from TCGA. IO-108 was discovered from a phage-displayed human single chain variable fragment antibody library. IO-108 contains the S228P mutation in the hinge region to prevent Fab-arm exchange. The binding affinity of IO-108 was measured using biolayer interferometry. The specificity of IO-108 was confirmed by two methods: 1) ELISA using recombinant LILR family members; 2) flow cytometry using cell lines engineered to express the extracellular domain of every LILR on the cell surface. Reporter and ligand binding assays were used to demonstrate LILRB2/ILT4 blocking activity of IO-108. Functional studies using primary immune cells from healthy donors and solid tumor cancer patients were performed to characterize IO-108 activity and mechanism of action. The in vivo efficacy of IO108 is currently being evaluated in mouse models.

Results We found high LILRB2/ILT4 expression associated with macrophage infiltration in many solid tumor types from 